\title{
Infrared Emission Spectra of Krypton and Argon
}

\author{
By Curtis J. Humphreys and Earle K. Plyler
}

\begin{abstract}
The analysis of the spectra of the noble atmospheric gases, utilizing descriptive data covering the photographically accessible region, has long indicated the possibility of a considerable extension of most of these spectra into the infrared region beyond 1.3 microns. Observations of the spectra of krypton and argon, in the region between 1 and 2 microns, have been made with a Perkin-Elmer spectrometer, fitted with a flint-glass prism cut to an angle of 55 degrees. The sources were Geissler tubes, used in previously reported work.

More than 15 new lines of krypton have been observed. Part of these are blends of unresolved pairs or groups. The emission maxima have been determined in favorable cases to a precision of two wave numbers, roughly equivalent to one-tenth of the smallest scale division on the wavelength drum. All observed lines have been classified, the most intense being represented by combinations of the type $2 p-3 d$, according to Paschen's notation. Two new levels from the configuration $s^{2} p^{5} f$ have been found. The remaining unobserved combinations of the type $1 s-2 p$, occurring in this region, are, with one exception, too weak to be observed. The argon infrared spectrum was observed by Paschen. More of its predicted combinations are in the photographic region than in the case of krypton. A few lines near 1.4 microns have been observed.
\end{abstract}

\section{Introduction}

The spectra originating in the neutral atoms of the noble atmospheric gases, neon, argon, krypton, and xenon, are among the most thoroughly studied of all line spectra observed and described to date. Examination of published data reveals very few lines of appreciable intensity that have not been fitted into a scheme of energy levels in accordance with the theory now universally accepted.

The first detailed and relatively complete analysis of any of the first spectra of the noble gases was that of neon, published by Paschen [1] ${ }^{1}$ in 1919. Paschen used a special notation to represent the spectral terms of neon. His system has been generally followed by other investigators reporting on the first spectra of the remaining noble gases, and is used in this paper.

The first spectrum of argon was classified by Meissner and reported in a series of papers begin-

\footnotetext{
1 Figures in brackets indicate the literature references at the end of this paper.
}

ning in 1926 [2]. In 1929 Gremmer [3] published the classification of the more prominent lines. He also extended the analysis of neon and argon in the photographically accessible infrared [4]. In 1929 also, Meggers, de Bruin, and Humphreys [5] published a detailed analysis of the first spectrum of krypton. Improved observations making available double the number of lines permitted a further extension of this classification in 1931 [6], with some minor revisions. A new paper by Gremmer [7] showed how all discrepancies between his analysis and that of Meggers, de Bruin, and Humphreys could be reconciled. Additional lines in the red and infrared were reported by Rasmussen [8].

The first spectrum of xenon was classified by Meggers, de Bruin, and Humphreys [9], with a subsequent revision and extension by Meggers and Humphreys [10]. Gremmer [11] also published an analysis in essential agreement with that of the authors just named. Rasmussen [8] 
extended the classification and contributed additional infrared data.

The above résumé of work on the first spectra of the noble gases is by no means complete. It includes references only to papers in which the analyses were brought to essentially their present form. The publications cited, however, give the complete history of the subject back to the discovery of these elements.

As soon as the energy-level systems for the noble gas atoms were fairly well established, it became evident that many relatively intense lines should occur in the infrared. The discovery of improved sensitizers for photographic plates has permitted a number of extensions of these spectra in the direction of greater wavelengths. Meggers and Humphreys published a paper on the infrared spectra of neon, argon, and krypton in 1933 [12]. Photographic observations as far as $12,000 \mathrm{~A}$ were reported. The paper also listed radiometric observations previously published by Paschen [13] on argon, and by Hardy [14] on neon. Complete term tables were given, incorporating the extensions permitted by the new data. These tables should be consulted in order to understand the discussion of the location of infrared lines as given in succeeding paragraphs. A later publication by Meggers [15] gives wavelengths and classifications of helium, neon, argon, krypton, and xenon lines as far as 13,000 A. These observations were made with Z-type Eastman plates and represent the present limit of photographic observations, utilizing silver halide emulsions incorporating photosensitizing dyes.

\section{Location of Infrared Emission Lines}

In conformity with generally observed characteristics of the spectra of homologous elements, there is a shift of analogous term combinations toward longer wavelengths as one goes from elements of lower to those of higher atomic number in the array of noble gases. In the first spectrum of neon most of the $1 s-2 p$ combinations are in the yellow, orange, and red regions and give the discharge its characteristic color. The $2 p-3 d$ combinations are in the photographically accessible infrared region, and appear with great intensity on plates treated with special sensitizers. The only combinations, in neon $\mathrm{I}$, out of the range of photography in the infrared, are those of the $3 d$ levels with the levels originating in the lowest $f$-electron configuration, and possibly as many as eight combinations of the type, $2 p-2 s$.

In argon I, fewer $1 s-2 p$ combinations are in the visible region than in neon $\mathrm{I}$, but all of them can be photographed. About half of the $2 p-3 d$ combinations are in the photographic range. The others are to be expected between 1.3 and $3 \mu$. The $d-f$ combinations involving lowest possible total quantum numbers are almost all just beyond the photographic region. Combinations of the type $2 p-2 s$ are in approximately the same region as $2 p-3 d$, and all the $2 s$ levels have been established by photographic observations.

The structure of krypton closely resembles that of argon with a somewhat greater displacement of analogous combinations toward greater wavelengths. Five of the $1 s-2 p$ combinations are beyond the range of photography, and extend as far as $2 \mu$. The $2 p-3 d$ combinations are predicted in the range between 1 and $10 \mu$, a few being of still greater wavelength. A number of these transitions have been observed photographically, namely, in instances where the $p$-level series converges to the lower, and the $d$-level to the higher ion limit. The $d-f$ combinations of first members of these series are, with a few exceptions, of a wavelength too great for photography. The second members of the $s$-series are of about the same magnitude as the first members of the $d$ series. This brings the $2 p-2 s$ combinations within the same range as those of the type $2 p-3 d$.

The predicted distribution of xenon lines in the infrared is considerably different from that of the other noble gases. Five $1 s-2 p$ combinations have not been observed. They are all too far out in the infrared for the radiation to be transmitted by the glass enclosures of the sources used in this work. They are also combinations between levels converging to different limits and not expected to be very intense. The $2 p$ and $3 d$ levels are of about the same magnitude, placing the combinations for the most part beyond the range of glass transmission. The interlimit transitions of the $2 p-3 d$ type fall mostly in the photographic range. This results from the very large difference between the ion limits amounting to $10,540 \mathrm{~cm}^{-1}$. The $d-f$ combinations fall in the photographic range. Only one of the $2 s$ levels is known.

It may be concluded that, of these spectra, krypton I, and argon 1, may be expected to have 
a considerable number of intense combinations between $1 \mu$ and $2 \mu$. Almost all intense neon lines may be photographed, whereas xenon has a very sparsely populated region between the photographic limit and about $5 \mu$. These considerations indicate that krypton and argon are the most promising of the noble gases for study in the region just beyond the limits of photography, but in which the glass enclosures of available sources are transparent.

\section{Observations}

The sources used in the earlier observations at the National Bureau of Standards were, for the most part, Geissler tubes, made by Robert Götze, in Leipzig, and of special construction permitting end-on illumination of the spectrometer slit. Several of these tubes were still in excellent condition, and were made available for these observations by the kindness of W. F. Meggers, of the Bureau's Spectroscopy Section.

A Perkin-Elmer model $12 \mathrm{~A}$ spectrometer, equipped with a General Motors amplifier and Brown recorder, was used as the dispersing, detecting, and recording system. Only slight modifications of this system were needed. The housing covering the projecting system was lifted, and the plane mirror rotated by a sufficient amount to permit forming on the entrance slit an image of the emission source, which was mounted beside the globar housing. The first recordings were made with the rock-salt prism in place. These served to establish the existence of the emission spectrum in the region between 1.3 and something over $2 \mu$. This, however, is near to the region of minimum dispersion for rocksalt, so that the conditions for wavelength measurement are unfavorable. Subsequently, a flint-glass prism cut to an angle of 55 degrees was mounted in the spectrometer. This angle was calculated to permit operating the instrument within the range of available wavelength dial settings without changing the setting of the Littrow mirror. By a proper selection of the prism angle, it is possible to interchange prisms of different materials without any readjustment of the other components, and to maintain the calibration for each prism.

Wavelength calibration was obtained by use of an H-4 mercury lamp. This is a source of high intensity and is steady in operation. The arc stream appears as a thin line when the source is warmed up, permitting a large concentration of energy in the slit opening. With this source it was possible to work with slit openings of $15 \mu$, and in some instances down to $12 \mu$. The mercury spectrum in the infrared is well known from the observations of McAlister [16], who used a glass multiprism spectrometer of high resolution, obtaining wave numbers that agreed remarkably well with those calculated from the known level scheme. The dial settings corresponding with the emission peaks of the mercury lines were determined from the averages of six recordings, and can be established to within one-tenth of a scale division. The mercury spectrum gives a good distribution of lines up to nearly $2 \mu$. The weakness of this array in a prismatic spectrum is that the four strongest lines at the longer wavelength end, namely, 1.6919, 1.6935, 1.7073, and $1.7108 \mu$, appear as two emission peaks, each consisting of an unresolved pair. The latter pair has a separation of $12 \mathrm{~cm}^{-1}$ in wave number and is just beyond the practical limit of resolution at this setting. The averages of the wave numbers of the respective pairs were adopted as the values for the observed peaks. It is expected that more satisfactory standards for the $2-\mu$ region can be found when more intense sources are available. Possibilities are the helium line at $2.058 \mu$ and the hydrogen line at $1.875 \mu$, the latter being the first line of the Paschen series. The wave numbers of the mercury lines were plotted against observed dial settings to give the calibration curve.

The noble gas sources were operated in the usual manner by connecting the tubes directly to the secondary of a sign transformer, rated at 12,000 v, 200 va. A variable-voltage transformer was placed in the primary to control the input voltage so as to prevent overloading. The energy output of these tubes was extremely small as compared with the mercury source. It was necessary in general to operate the amplifier gain setting as high as possible. This required unusually good operating conditions in the room because very slight temperature changes caused a large amount of zero drift. The slit settings were kept as small as possible, consistent with reasonable deflections, but in general it was necessary to operate in the range of slit-width settings between 60 and $120 \mu$. Repeated runs were made with different amplifier gains and different slit widths, chosen to give the best record of characteristic features. Thus the 
most intense lines could be observed with narrower slits and lower amplifier gains, whereas it was necessary to increase both to the practical limit in order to obtain the faintest lines. It was encouraging to find, however, that the sharpest maxima could be determined with a precision of about one-tenth of a scale division from repeated records. The less well-defined maxima are probably fixed with a precision about half as great. At $6,000 \mathrm{~cm}^{-1}$, a scale division corresponds to about 15 wave numbers, when the glass prism is in place.

The portions of the red and infrared spectra of krypton and argon falling within the range of photographic observation have been reobserved by the methods reported in this paper. The wavelengths of the lines so observed were found to conform with the system of mercury standards and with the noble gas lines of lesser wavelength. Inasmuch as these lines have already been observed with a precision that could not be matched with the radiometric technic used in this work, they are not listed in this paper except where classified on the basis of new observations.

\section{Discussion of Results}

The results obtained for krypton, together with their interpretation, are shown in table 1 . Here are shown observed wave numbers and corresponding wavelengths, estimated intensities on a scale 1 to 10 , calculated wave numbers, and interpretation as combinations of levels. The first two lines entered in the table were observed, but, inasmuch as the calibration curve obtained from mercury lines cannot be extrapolated with reasonable accuracy beyond about $1.7 \mu$, only the wave numbers, computed from known level values, are listed.

The surprising feature of this spectrum is the failure of the previously unobserved $1 s-2 p$ combination to appear with appreciable intensity. The line with adopted wave number 5,322 classified $1 s_{2}-2 p_{10}$ is the only one of this group appearing with sufficient intensity to be observed by the Bureau's present equipment.

The most intense krypton lines observed are combinations of the type, $2 p-3 d$. These can be classified both on the basis of computed wave numbers and the intensities of the higher members of the respective series, all of which are observed photographically. The fact that the combinations $2 p_{8}-3 d_{4}$ and $2 p_{9}-3 d_{4}^{\prime}$ show the highest intensities among this group is in accordance with expectations. The line observed at $5,930 \mathrm{~cm}-{ }^{1}$ is much stronger than any of the others. As noted in the table, it is possible that five different lines may contribute to this maximum. Considering the slit widths that were required, it is probable that the limit of resolution when using the noble gas sources was not better than about $25 \mathrm{~cm}^{-1}$, so that these ambiguities cannot be avoided until the technic can be improved.

TABLE 1.-List of $\mathrm{Kr}$ I lines

\begin{tabular}{|c|c|c|c|c|}
\hline Intensity & $\lambda_{\mathrm{obs}}$. & $\nu_{\text {obs }}$. & Term combination & $\nu_{c a l e}$. \\
\hline & Microns & $\mathrm{cm}^{-1}$ & & $\mathrm{~cm}^{-1}$ \\
\hline 1. & - n & - n & $1 s_{2}-2 p_{10}$ & 5,322 \\
\hline 5 & - & - & $2 p_{9}-3 d_{4}^{\prime}$ & 5,503 \\
\hline 1. & 1. 7820 & 5,610 & $2 p_{10}-3 d_{6-}$ & 5,603 \\
\hline \multirow[t]{3}{*}{$1 \ldots \ldots$} & 1. 7347 & 5,763 & $2 p_{2}-3 s_{1}^{\prime \prime \prime}$ & 5,756 \\
\hline & & & $\left(2 p_{s-3}-3 d_{4-}\right.$ & 5,919 \\
\hline & & & $2 p_{9}-3 d_{4} \ldots$ & 5,932 \\
\hline \multirow[t]{3}{*}{$10 \ldots$} & 1. 6859 & 5,930 & $2 p_{10}-3 d_{5}$ & 5,917 \\
\hline & & & $2 p_{7}-3 d_{1-}^{\prime \prime}$ & 5,903 \\
\hline & & & $2 p_{6}-3 d_{1-.}^{\prime}$ & 5,956 \\
\hline \multirow{2}{*}{1.} & 1.5823 & 6,318 & $\int 3 d_{2}-4 \mathrm{X} \ldots$ & 6,319 \\
\hline & 1.5020 & 0,010 & $3 d_{2}-4 Z_{\ldots}$ & 6,320 \\
\hline \multirow{3}{*}{4.} & 1.5326 & 6.523 & $\int 2 p_{10}-3 d_{3 \ldots}$ & 6,519 \\
\hline & & & $\mid 2 p_{6}-3 d_{2}$ & 6,522 \\
\hline & & & $22 p_{9}-3 d_{1--}^{\prime}$ & 6,785 \\
\hline \multirow[t]{3}{*}{3} & 1.4747 & 6,779 & $\left\{2 p_{8}-3 d_{1-\ldots}^{\prime}\right.$ & 6,772 \\
\hline & & & $2 p_{6}-3 s_{4} \ldots$ & 6,771 \\
\hline & & & $\int 2 p_{7}-2 s_{4-}$ & 6,930 \\
\hline \multirow[t]{2}{*}{3} & 1. 4424 & 6,931 & $\left\{2 p_{2}-2 s_{2} \ldots \ldots\right.$ & 6,942 \\
\hline & & & $3 d_{1}^{\prime}-4 \mathrm{X}$ & 6,942 \\
\hline 2 & 1. 3926 & 7,179 & $3 d_{1}^{\prime \prime}-4 W_{\ldots}$ & 7,180 \\
\hline \multirow[t]{2}{*}{6} & 1. 3631 & 7,334 & $\left\{2 p_{8}-3 d_{2} \ldots \ldots\right.$ & 7,338 \\
\hline & & & $\left|2 p_{9}-2 s_{5-\ldots}\right|$ & 7,333 \\
\hline 2 & 1. 3203 & 7,572 & $2 p_{8}-2 s_{4} \ldots$ & 7,587 \\
\hline 2 & 1. 2883 & 7,760 & $3 d_{4}-4 U_{\ldots} \ldots \ldots$ & 7762.5 \\
\hline & a1. 220454 & 8191.43 & $3 d_{4}^{\prime}-4 U_{\ldots} \ldots \ldots$ & 8191.43 \\
\hline & a1. 211781 & 8250.06 & $3 d_{4}^{\prime}-4 \mathrm{~W} \ldots \ldots$ & 8250.06 \\
\hline
\end{tabular}

a Wave lengths given by W. F. Meggers.

There are a number of combinations of the type $2 p-2 s$. However, there is still no evidence for the missing $2 s_{3}$ term. As it has not been found from photographic data, only a combination involving $2 p_{3}$ or $2 p_{4}$ could reveal it.

The remaining lines are classified as combinations of $f$-type levels. Two of these designated, $4 \mathrm{U}$ and $4 \mathrm{~W}$, are new. It is interesting to note that they explain two previously unclassified lines given by Meggers, at wave numbers 8250.06 and 8191.43. The level values, in accordance with the latest revision of the krypton term table 
[12], and respective inner quantum numbers are as follows:

$$
\begin{aligned}
& 4 \mathrm{U}, 6926.04, j=4 \\
& 4 \mathrm{~W}, 6867.41, j=3 .
\end{aligned}
$$

The experimental evidence for these new levels is the appearance of two constant differences, the first, between the lines 8191.43 and 7,760 $\mathrm{cm}^{-1}$, equal to the difference between $3 d_{4}^{\prime}$ and $3 d_{4}$, and the second, between the lines 8,250.06 and $7,179 \mathrm{~cm}^{-1}$, equal to the difference between $3 d_{4}^{\prime}$ and $3 d_{1}^{\prime \prime}$. Each of these differences established a new level. Additional evidence that removes any doubt as to the reality of these levels, is that extrapolation of the hitherto incomplete series, designated $\mathrm{U}$ and $\mathrm{W}$, back to their respective first members, gives values practically identical with those experimentally determined for the two new levels. It becomes apparent therefore, that these levels are the first members of the $\mathrm{U}$ and $\mathrm{W}$ series, and the reason for the choice of designation is obvious. These $f$-type series are nearly hydrogenic and can be readily extrapolated by means of a Rydberg table [17].

Very few new data were obtained for the argon spectrum, since most of it is in the photographically accessible region. Two of the lines observed by Paschen, 7402.9 and $7287.3 \mathrm{~cm}^{-1}$, are those of greatest wavelength to appear with appreciable intensity. An emission peak at $7,477 \mathrm{~cm}^{-1}$ is probably $2 p_{6}-3 d_{1}^{\prime}$, or $2 p_{2}-2 s_{2}$ or a blend of these.

\section{Conclusion}

The infrared measurements reported in this paper are to be regarded as preliminary. The investigation was intended to demonstrate the feasibility of exciting these spectra with sufficient intensity for observation, and of operating with available apparatus and methods. Work is in progress on the design and construction of new apparatus for study of line emission spectra in the infrared. The principal unit is to be a grating spectrometer equipped with a grating ruled to concentrate the radiant energy in a selected region. Several such gratings will be available. A second improvement that must be made is the utilization of sources permitting very much greater concentration of incident radiation at the entrance slit of the spectrometer.

\section{References}

[1] F. Paschen, Ann. Physik [4] 60, 405 (1919).

[2] K. W. Meissner, Z. Physik. 37, 238 (1926); 39, 172 (1926); 40, 839 (1927).

[3] W. Gremmer, Z. Physik. 54, 199 (1929).

[4] W. Gremmer, Z. Physik. 50, 721 (1928).

[5] W. F. Meggers, T. L. deBruin, and C. J. Humphreys, BS J. Research 3, 129 (1929) RP89.

[6] W. F. Meggers, T. L. deBruin, and C. J. Humphreys, BS J. Research $\boldsymbol{7}, 643$ (1931) RP364.

[7] W. Gremmer, Z. Physik. 73, 620 (1932).

[8] E. Rasmussen, Z. Physik. 73, 779 (1932).

[9] W. F. Meggers, T. L. deBruin, and C. J. Humphreys, BS J. Research 3, 731 (1929) RP115.

[10] C. J. Humphreys and W. F. Meggers, BS J. Research 10, 139 (1933) RP521.

[11] W. Gemmer, Z. Physik. 59, 154 (1930).

[12] W. F. Meggers and C. J. Humphreys, BS J. Research 10, 427 (1933) RP540.

[13] F. Paschen, Ann. Physik [4] 27, 537 (1908).

[14] J. D. Hardy, Phys. Rev. 38, 2162 (1931).

[15] W. F. Meggers, Zeeman Verhandelingen, p. 190 to 200 (Martinus Nijhoff, The Hague, 1935); J. Research NBS 14, 487 (1935) RP781.

[16] E. D. McAlister, Phys. Rev. 34, 1142 (1929).

[17] Rydberg Interpolation Table (Departments of Physics and Astronomy, Princeton University, Princeton, N. J., 1934).

Washington, JANuARy 23, 1946. 